\title{
Large Eddy Simulation of Autoignition in a Turbulent Hydrogen Jet Flame Using a Progress Variable Approach
}

\author{
Rohit Kulkarni and Wolfgang Polifke \\ Lehrstuhl für Thermodynamik, TU München, Boltzmannstraße 15, Garching, Germany \\ Correspondence should be addressed to Rohit Kulkarni, kulkarni@td.mw.tum.de \\ Received 27 October 2011; Accepted 4 January 2012 \\ Academic Editor: Vladimir A. Sabel'nikov
}

Copyright ( $) 2012$ R. Kulkarni and W. Polifke. This is an open access article distributed under the Creative Commons Attribution License, which permits unrestricted use, distribution, and reproduction in any medium, provided the original work is properly cited.

\begin{abstract}
The potential of a progress variable formulation for predicting autoignition and subsequent kernel development in a nonpremixed jet flame is explored in the LES (Large Eddy Simulation) context. The chemistry is tabulated as a function of mixture fraction and a composite progress variable, which is defined as a combination of an intermediate and a product species. Transport equations are solved for mixture fraction and progress variable. The filtered mean source term for the progress variable is closed using a probability density function of presumed shape for the mixture fraction. Subgrid fluctuations of the progress variable conditioned on the mixture fraction are neglected. A diluted hydrogen jet issuing into a turbulent coflow of preheated air is chosen as a test case. The model predicts ignition lengths and subsequent kernel growth in good agreement with experiment without any adjustment of model parameters. The autoignition length predicted by the model depends noticeably on the chemical mechanism which the tabulated chemistry is based on. Compared to models using detailed chemistry, significant reduction in computational costs can be realized with the progress variable formulation.
\end{abstract}

\section{Introduction}

Autoignition in nonpremixed and partially premixed turbulent flow is of interest for industrial applications such as sequential gas turbines or HCCI (Homogeneously Charged Compression Ignition) piston engines, see [1] for a recent review. The present work is motivated by the need for a more accurate model of reheat combustion in sequential gas turbine combustors $[2,3]$. In a sequential gas turbine system, hot gas produced in a first combustion chamber expands through a high pressure turbine before additional fuel is injected in a second "reheat combustion chamber." Since highly preheated exhaust gases enter the reheat combustor, autoignition plays an important role in determining flame position and thus emissions.

A characteristic of autoignition is an induction period, during which a pool of radicals is built up without significant heat release. During that phase, temperatures are typically rather low, such that steady-state or partial equilibrium relations may not be invoked to reduce the chemical mechanism. The induction period is followed by a fast heat release phase, during which the intermediate radicals from the radical pool are consumed rapidly and combustion products are formed. If autoignition occurs in turbulent flows, the chemical and the fluid-mechanical time scales are often of the same order. Simplifying assumptions like fast (or slow) chemistry, which are frequently exploited in combustion modelling, are not valid in that case.

In nonpremixed cases, the reaction progress during the induction period depends in a sensitive manner on the fuel/ oxidizer composition, that is, the mixture fraction. This is in particular so if the fuel and oxidizer streams exhibit different temperatures, which is the case, for example, in reheat gas turbine combustion [2]. The chemical reactions are fastest at the so-called "most-reactive mixture fraction," which may differ from the stoichiometric one [1]. The mixing between fuel and oxidizer streams is thus of crucial importance in determining the location of autoignition. Turbulence influences the mixing and reaction in a significant and nontrivial manner. In general, more intense turbulence enhances mixing and thus reduces the autoignition length. However, excessive turbulence may dilute the radicals with the reactants 
at a faster rate, resulting in longer ignition delays or even suppression of autoignition. In order to predict ignition correctly, a combustion model must consider the interactions between chemical and fluid-mechanical phenomena in an adequate manner.

In this work, the experiment of Markides and Mastorakos [4] on hydrogen autoignition in a turbulent coflow was chosen as a test case for an LES combustion model, which combines a composite progress variable with a probability density function (PDF) (in the LES context, the term "filtered density function (FDF)" is also used.) of presumed shape for the mixture fraction. Previously, this configuration was modelled with Conditional Moment Closure (CMC) by Mastorakos et al. [5] and Stanković et al. [6]. It is argued in [7] that amongst the presumed PDF methods, CMC is most adequate for predicting autoignition. In the $\mathrm{CMC}$ formulation, transport equations are derived for reactive species conditioned on mixture fraction and solved on a coarse CMC grid. The method rests on the assumption that the gradient of conditioned values of the reactive species is much weaker in physical space than in mixture fraction space. The fluctuations of the reactive species conditioned on the mixture fraction may thus be neglected (assumption of "Presumed Conditional Moments", PCM [8]).

The stochastic fields method, which is a transported PDF method based on an Eulerian approach, was also found to predict successfully autoignition and subsequent flame behavior for the experiment of Markides and Mastorakos [4]. Both the CMC and the stochastic fields methods used detailed chemical mechanism. Indeed, it has been argued [7] that the use of detailed chemistry is necessary for accurate prediction of autoignition. However, this implies very high computational costs, due to the large number of transport equations that need to be solved with detailed chemistry.

A significantly less expensive method based on a progress variable and a presumed PDF to represent subgrid fluctuations has been formulated by [9-12]. This model reduces the detailed chemistry to two dimensions, namely, the mixture fraction and a progress variable, thus minimizing the number of transport equations to be solved. In order to properly describe the buildup of radicals during the induction phase of autoignition, the progress variable is defined as a combination of an intermediate and a product species. The dependence of the rate of reaction progress on mixture fraction and progress variable is determined in a preprocessing step from spatially homogeneous 0D reactor computations with detailed chemistry. This model was applied to the target experiment by Galpin et al. [13] for high coflow temperature $(1000 \mathrm{~K})$. In the present work, the potential of PV-PPDF (Progress Variable-Presumed Probability Density Function) model to simulate transient phenomena like autoignition and subsequent flame propagation in LES context for temperatures lower than those considered in [13] is investigated. The lower temperature range is challenging as a broader range of Damköhler numbers has to be considered due to slower chemistry.

The paper is organized as follows: in the next section, the model formulation is presented. Then the experimental setup of Markides and Mastorakos [4], used for model validation, is described. After outlining briefly the numerical setup for validation studies, model results are compared against measurements.

\section{Model Formulation}

Before presenting the model formulation in detail, pertinent prior work is reviewed briefly. For turbulent premixed flames, Bradley and coworkers [14] suggested a "laminar flamelet model," where the PDF of a reaction progress variable based on temperature rise is approximated by a beta function. Mean values of reaction progress and chemical species are obtained by integration over a detailed chemical kinetic laminar flame structure, weighted with the presumed PDF of the reaction progress variable. The influence of turbulent strain on mean reaction progress was taken into account by considering strained laminar flames and/or extinction at a critical strain rate [15]. In the mid90s, this approach was used at ABB to model (partially) premixed combustion in gas turbines, including the case of nonhomogeneous mixture fraction [16, 17]. A similar approach for modelling autoignition under high pressure Diesel engine conditions was proposed by Chang et al. [18]. A flamelet library for the source term of the progress variable with 5 dimensions (progress variable, mixture fraction mean, mixture fraction variance, scalar dissipation rate, and pressure) was built in the preprocessing step. The source term for the progress variable was weighted with the presumed beta-PDF shape of the reaction progress variable $\left(\mathrm{CO}_{2}\right)$.

The flamelet model with lookup table was generalized to more than one progress variable by De Goey and Ten Thije Boon Kkamp [19], who coined the popular term "flamelet generated manifolds." An adaptation to autoignition in a turbulent jet-in-cross-flow configuration, as it is found in reheat combustors, was proposed by Brandt et al. [3, 20, 21], with a progress variable based on intermediate species formed during the induction period. The lookup table was built from $0 \mathrm{D}$ reactors with detailed chemistry, rather than reactivediffusive structures. For large eddy simulation of partially premixed or nonpremixed flames, formulations based on progress variable and mixture fraction were proposed by Pierce and Moin $[9,10]$ and Vervisch and coworkers $[8,11$, 12].

In the present paper, the model of Brandt et al. [3, 20, 21 ] is developed further. Firstly, a progress variable based on a combination of intermediate and final product species is introduced, such that it describes both the buildup of radical species during the induction period and the subsequent heat release. Secondly, following [9-12] the model is implemented in the LES context.

2.1. Definition and Tabulation of Progress Variable. Both the buildup of radical species during the induction period, which is typically comparatively slow, and the rapid conversion of fuel and oxidizer to final products after ignition control the distribution of heat release in autoignition combustion. Accordingly, a progress variable for autoignition should be defined as a combination of intermediate and final 


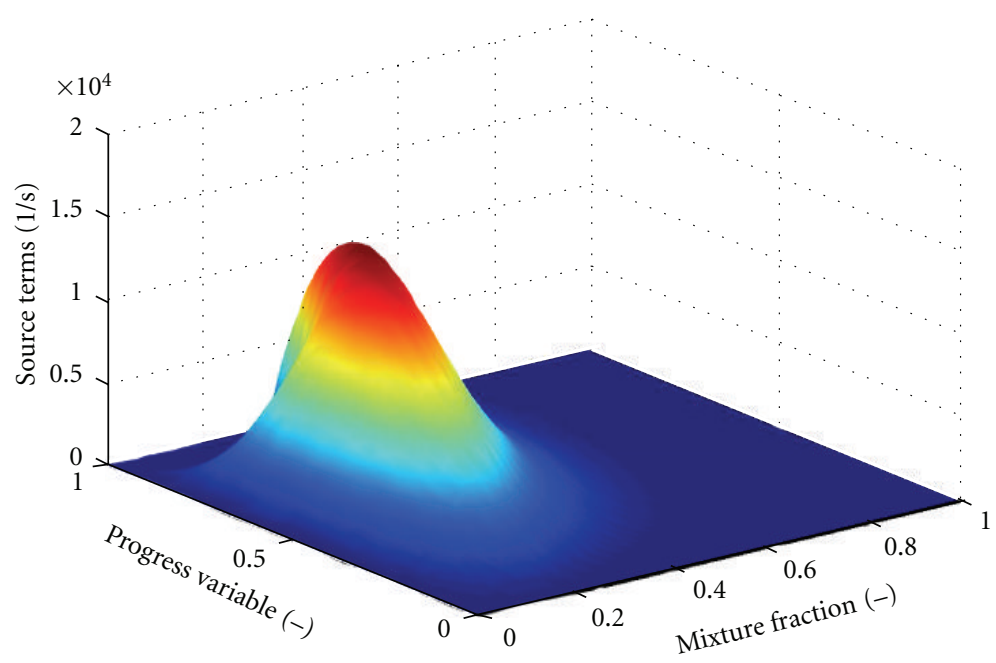

(a)

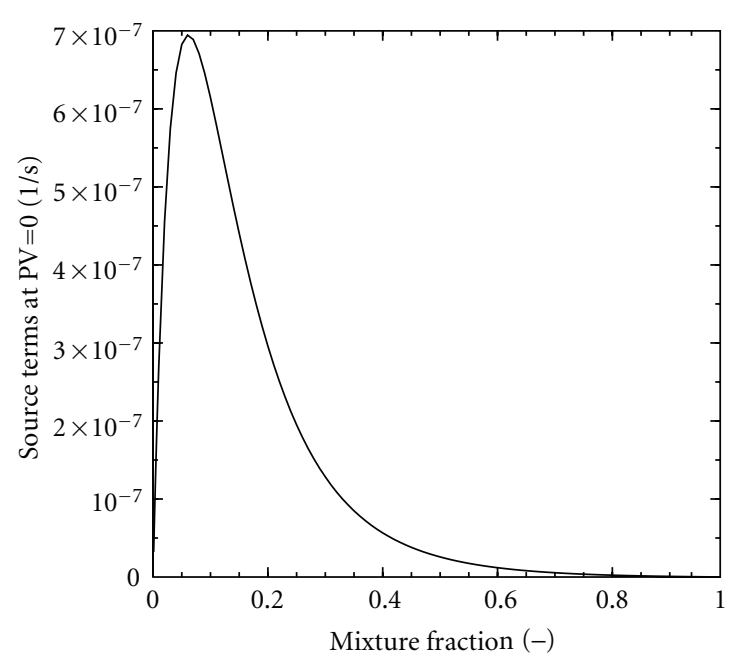

(b)

FIGURE 1: (a): progress variable source term $\dot{\omega}_{Y_{c}}$ versus mixture fraction $Z$ and normalized progress variable PV for Case 0 computed with Ó Conaire mechanism. (b): initial PV source term $(\mathrm{PV}=0.0)$. Boundary conditions: Case 0 described in Table 1.

TABle 1: Simulated cases.

\begin{tabular}{lcccc}
\hline Case & Air temperature $[\mathrm{K}]$ & Fuel temperature $[\mathrm{K}]$ & Bulk velocity $[\mathrm{m} / \mathrm{s}]$ & Observation \\
\hline 0 & 950 & 750 & 26 & Random Spots \\
1 & 955 & 750 & 26 & Random Spots \\
2 & 960 & 750 & 26 & Random Spots \\
3 & 980 & 750 & 26 & Flashback \\
\hline
\end{tabular}

product species. When doing so, one must ensure that for given mixture fraction, the progress variable $Y_{c}$ increases monotonously from initial conditions up to equilibrium.

In this work, autoignition of hydrogen is considered, so $\mathrm{H}_{2} \mathrm{O}$ is an obvious choice for the product species. The intermediate species $\mathrm{HO}_{2}$ is included in the definition of the progress variable to represent the radical pool, which builds up due to "slow chemistry" during the induction phase, before significant generation of combustion products. A composite progress variable may be defined as the sum of the mass fractions of $\mathrm{H}_{2} \mathrm{O}$ and $\mathrm{HO}_{2}$ :

$$
Y_{c}=Y_{\mathrm{HO}_{2}}+Y_{\mathrm{H}_{2} \mathrm{O}}
$$

The normalized progress variable (PV) is defined as the ratio of the mass fraction of the composite progress variable divided by its equilibrium value. Equation (2) defines the normalized progress variable (PV) used for the definition of the ignition criteria in this work:

$$
\mathrm{PV}=\frac{Y_{\mathrm{HO}_{2}}+Y_{\mathrm{H}_{2} \mathrm{O}}}{Y_{\mathrm{HO}_{2}, \mathrm{eq}}+Y_{\mathrm{H}_{2} \mathrm{O}, \mathrm{eq}}} .
$$

The source term $\dot{\omega}_{Y_{c}}$ for the progress variable $Y_{c}$ must be computed with detailed chemistry in a preprocessing step and tabulated as a function of the parameters that control reaction progress. With the "steady laminar flamelet" approach as originally proposed by Bradley and coworkers $[14,15]$, these parameters are progress variable $Y_{c}$, possibly complemented by mixture fraction $Z$ and flame strain $a$ or scalar dissipation $\chi$. Detailed chemistry data for (partially) premixed or nonpremixed combustion are thus typically generated by solving 1-dimensional reactive-diffusive model configurations, for example, propagating or strained flames.

However, the radical buildup during the induction period, which is comparatively slow and not associated with significant heat release, does not lead to formation of thin reactive-diffusive structures. Therefore, in this work spatially homogeneous, transient $0 \mathrm{D}$ reactor computations with detailed chemistry were used to generate lookup tables for the source term $\dot{\omega}_{Y_{c}}\left(Z, Y_{c}\right)$. Brandt et al. $[3,20]$ used a similar approach, but considered only intermediate species up to a maximum concentration, such that heat release could not be captured.

Figure 1 shows typical results obtained with the mechanism for hydrogen oxidation of Ó Conaire et al. [22] ("Ó Conaire mechanism" in the following). The boundary conditions for the look-up table generation is described in Table 1 (Case 0) and the method to generate the lookup table are described in Section 5. On Figure 1(b), the initial source term $(\mathrm{PV}=0.0)$ is shown as a function of mixture fraction. It is interesting to note that the source term is not zero at $\mathrm{PV}=$ 0.0 . This is the reason why at low temperature conditions, where steady-state or partial equilibrium assumptions may not be invoked, the concept of chemistry tabulation based on mixture fraction and progress variable was shown to perform better than the ILDM method [23]. The source term 
is maximum for leaner mixtures $Z<Z_{\text {st }}\left(Z_{\text {st }}=0.17\right)$ due to mixing temperatures above those of the stoichiometric case. It will be shown later (Section 5) using 0D premixed reactors that the minimum ignition delay is observed at a leaner mixture fraction called as "most reactive." Figure 1(a) shows that as the reactions progress (increase in PV), the source terms increase nonlinearly. The maximum source term is found near the stoichiometric mixture fraction $\left(Z_{\text {st }}=0.17\right)$. Form this information, it can be inferred that at initial stages (preignition), the chemical reactions are faster at leaner mixture fraction, whereas at later stages (heat release), the chemical reactions surrounding the stoichiometric mixture fraction are faster.

2.2. Transport Equations and LES Model. Transport equations are solved for the filtered mixture fraction $\tilde{Z}(3)$ and the filtered progress variable $\widetilde{Y}_{c}(4)$

$$
\bar{\rho} \frac{\partial \tilde{Z}}{\partial t}+\bar{\rho} \tilde{u} \cdot \nabla \tilde{Z}=-\nabla \overline{\tau_{Z}}+\nabla \cdot(\bar{\rho} D \nabla \tilde{Z}),
$$

$$
\bar{\rho} \frac{\partial \widetilde{Y_{c}}}{\partial t}+\bar{\rho} \tilde{u} \cdot \nabla \widetilde{Y_{c}}=-\nabla \overline{\tau_{Y_{c}}}+\nabla \cdot\left(\bar{\rho} D \nabla \widetilde{Y_{c}}\right)+\bar{\rho} \tilde{\dot{\omega}}_{Y_{c}} \cdot
$$

The source term $\dot{\omega}_{Y_{c}}$ for the filtered reaction progress variable is unclosed and requires modelling. Two approaches are frequently employed, namely, the "transported PDF" and the "presumed PDF" methods. The transported PDF method samples over possible subgrid realizations and needs modeling only for the subgrid or molecular mixing term. The major advantage of this method is that the chemical source term is closed. The major disadvantage of the stochastic method is the high computational cost, which hinders the application to configurations of applied interest.

In the presumed PDF methods, transport equations are solved for the statistical moments, like the mean and variance, of relevant flow variables. Closure for the chemical source terms is achieved by presuming the functional form of the PDF, which is parametrized in terms of the moments. In the case of binary mixing, a beta PDF shape is most commonly used for the mixture fraction. For multivariate PDFs, the definition of a suitable functional form remains a challenge [21].

In this work, a beta function is assumed for the probability distribution of the mixture fraction in order to achieve closure for the reaction source term

$$
\tilde{\omega}_{Y_{c}}=\int_{0}^{1} \dot{\omega}_{Y_{c}}\left(Z,\left(Y_{c} \mid Z\right)\right) \tilde{P}(Z) d Z
$$

The conditioned value of progress variable, which appears in the above equation, is calculated according to [8]

$$
Y_{c} \mid Z \approx\left(\frac{\widetilde{Y}_{c}}{\widetilde{Y}_{c}^{\mathrm{Eq}}}\right) Y_{c}^{\mathrm{Eq}}(Z)
$$

This assumption, called PCM, presumes the conditional moment without solving the transport equations for it [8].
Indeed, the progress variable concept is sometimes referred to as "Simplified version of CMC" [8], in the sense that the conditional moments are presumed rather than calculated. In comparison to the CMC model, in the progress variable approach, transport equations are solved for the progress variable rather than for all the species from the detailed chemical mechanism conditioned on the mixture fraction. This is the reason for the reduced computation cost of the progress variable approach compared to the CMC model. It is to be noted that the subgrid fluctuations of the progress variable are not completely neglected, but the fluctuations conditioned on the mixture fraction are neglected. The similarity between the model described above and the CMC model is that the conditioned fluctuations about the mixture fraction are neglected and a presumed shape of the mixture fraction is used to close the chemical source term.

Mixture fraction variance was modeled using a dynamic model suggested by Pierce and Moin [24]

$$
Z^{\prime \prime 2}=C_{v} \Delta^{2}|\nabla \tilde{Z}|^{2}
$$

where $C_{v}$ is the Smagorinsky constant.

Model assumptions may be summarized as follows

(1) Reaction progress is described by a single progress variable. For the case of hydrogen autoignition, the progress variable is based on concentrations of intermediate $\mathrm{HO}_{2}$ and product species $\mathrm{H}_{2} \mathrm{O}$.

(2) No thin reaction-diffusion structures are formed during the induction period, thus a direct impact of strain rate or scalar dissipation on reaction progress is neglected.

(3) The subgrid fluctuations of the progress variables conditioned on the mixture fraction are neglected.

(4) The shape of probability density function for mixture fraction fluctuations is presumed (Beta PDF).

(5) Differential and preferential diffusion effects are not considered.

\section{Experimental Configuration}

Figure 2 shows the experimental setup of the test case [4]. In the experiment, diluted hydrogen ( $13 \%$ by mass) is injected coaxially into a hot flow of air through a thermally insulated quartz tube. The air is heated electrically and enters into the quartz tube through a perforated plate $(3.0 \mathrm{~mm}$ holes and $44 \%$ blockage) to promote turbulence. The integral length scales and the turbulent intensities were measured in the coflow at the injector plane. The bulk velocity of air is controlled at $26 \mathrm{~m} / \mathrm{s}$. Diluted hydrogen (13\% hydrogen and $87 \%$ nitrogen by mass) is passed through a $2.25 \mathrm{~mm}$ injector at $26 \mathrm{~m} / \mathrm{s}$. The temperature of air and the velocity of the fuel were varied to obtain different autoignition regimes. $\mathrm{OH}$ chemiluminescence was used in the experiments to determine the autoignition lengths. Four different regimes were observed depending on the coflow temperature. The "noignition" regime was observed at very low temperatures, followed by "random spots regime," where ignitions kernels 


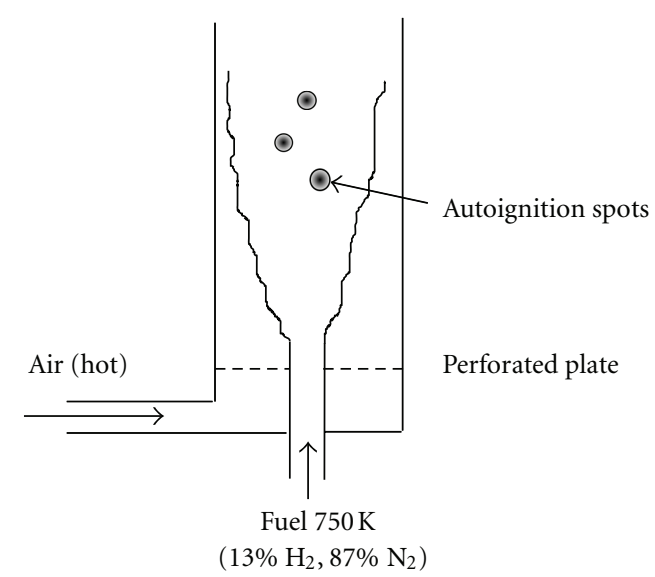

Figure 2: Schematic of the experimental setup [4].

occur sporadically and are transported out of the tube. The third regime is "flashback," in which autoignition occurs downstream of injection, and then the flame propagates upstream towards the injector, resulting in an anchored or lifted flame. The fourth regime is the diffusion flame. In this regime, the fuel and oxidizer react as soon as they come in contact.

\section{Numerical Setup}

The computations were carried out using the FLUENT LES solver [25]. The dynamic Smagorinsky-Lilly model was used as a subgrid model. Although the length of the quartz tube in the experiment is $0.5 \mathrm{~m}$, in this study a length of $0.15 \mathrm{~m}$ was used, as it is sufficiently long for the cases considered here. Adiabatic boundary conditions were imposed at the walls. To reproduce the turbulence generated by the grid, the synthetic turbulence generator model available in FLUENT was used, with $4 \mathrm{~mm}$ turbulence length scale and 14\% turbulence intensity. The temperatures and the bulk velocities for the different cases are shown in Table 1 . The bulk velocity of the coflow and fuel was $26 \mathrm{~m} / \mathrm{s}$ and the temperature of the fuel is $750 \mathrm{~K}$. The simulations were carried out with a constant time step of $1 e-6 \mathrm{~s}$, which corresponds to a Courant number less than unity. The required CPU time of simulation is $520 \mathrm{CPU}$ hours (QuadCore Computer: 150 hours). For comparison, the simulation time reported by [7] using the stochastic fields method with detailed chemistry was about 4000 CPU hours. The mesh size in that study was comparable to the one used in the present work. The reduction of the computational cost is a major advantage of the progress variable approach for industrial applications.

\section{Results and Discussion}

5.1. OD Reactor Calculations. For combustion modelling, and perhaps in particular for prediction of autoignition, the chemical kinetic mechanism is crucially important. When validating or comparing turbulence-chemistry interaction models, the same chemical mechanism should be used, if possible, or the impact of the chemical mechanism on modeling results should be appraised. In this study, the mechanisms of Yetter et al. [26] and Ó Conaire et al. [22] were first compared to each other in 0D calculations with CANTERA [27] for the ignition delay and were then used to tabulate the chemical source term.

The mechanism of Yetter et al. [26], which is validated for $\mathrm{CO}$ and $\mathrm{H}_{2}$ fuels over a wide range of temperature, was used previously by Navarro-Martinez and Jones [7] for LES of the case considered in this study. Stanković et al. [6] compared different chemical mechanisms with respect to autoignition delays and used the Li mechanism [28] for LES. In that study, the ignition delays predicted by the Li mechanism and the O Conaire mechanism were found to be similar, but significant discrepancies with the Yetter mechanism were observed. The $\mathrm{Li}$ and the Ó Conaire mechanisms have been validated for $\mathrm{H}_{2}$ combustion on a wide range of temperature conditions. Hence, in order to validate the model based on the previous LES studies, both the Yetter and the Ó Conaire mechanisms were used in this work. Zero-dimensional plug flow reactor calculations (only time dimension) were performed in order to compare the two chemical mechanisms. The calculations were done for all the four cases over a range of mixture fractions. In Figure 3 the ignition delays predicted by both the mechanisms are compared. The most reactive mixture fraction is also shown.

Figure 3 shows that the ignition delay predicted by the Yetter mechanism is shorter than that by the Ó Conaire mechanism. Hence, it is expected from LES that the autoignition length predicted by the Yetter mechanism in LES will be shorter than that by the Ó Conaire.

5.2. LES Results. In this section LES results for the cases listed in Table 1 will be presented. After the mesh quality study, the mixing field will be described first, followed by the autoignition location.

5.2.1. LES Mesh Quality. To study the LES mesh quality, two meshes with different sizes were used. Mesh 1 comprises of $150 \times 70 \times 48$ nodes in axial, radial, and azimuthal directions respectively. The corresponding number of nodes for a finer mesh, Mesh 2, are $200 \times 90 \times 56$. The mesh was clustered towards the central axis in order to resolve the mixing layer between the fuel and the air stream. Case 0 boundary conditions were used for this study.

The following LES quality index suggested by [29] based on the viscosity ratio has been used for the study:

$$
\mathrm{LES}_{-} \mathrm{IQ}=\frac{1}{1+0.05\left(\nu_{\mathrm{t}, \mathrm{eff}} / \nu\right)^{0.53}} .
$$

In $(8), v_{t, \text { eff }}$ is the effective viscosity (laminar and turbulent) and $v$ is the laminar viscosity. According to [29], the LES quality index should be above 0.8 for quality LES mesh.

The contour plots on Figure 4(a) show snapshots the LES quality index for both the meshes. The LES quality criteria based on the above index has been satisfied by both the meshes. Mesh 2 has higher values of the index suggesting a better quality due to more refined mesh. 


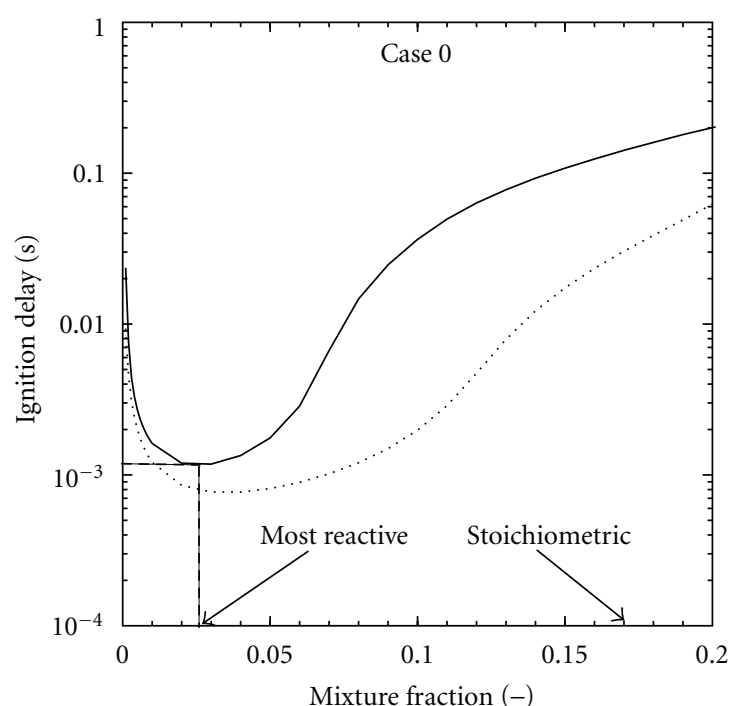

(a)

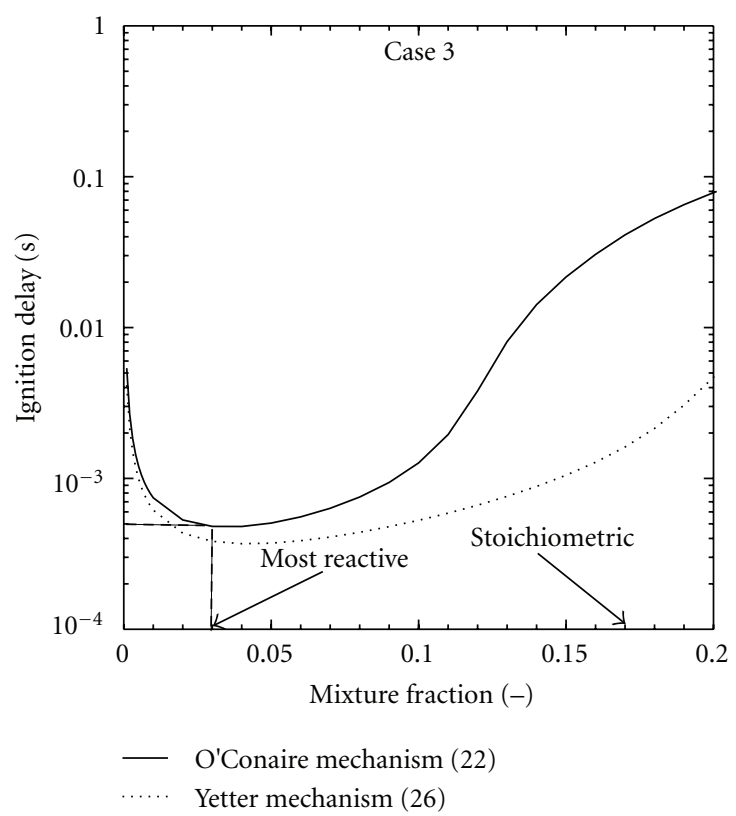

(c)

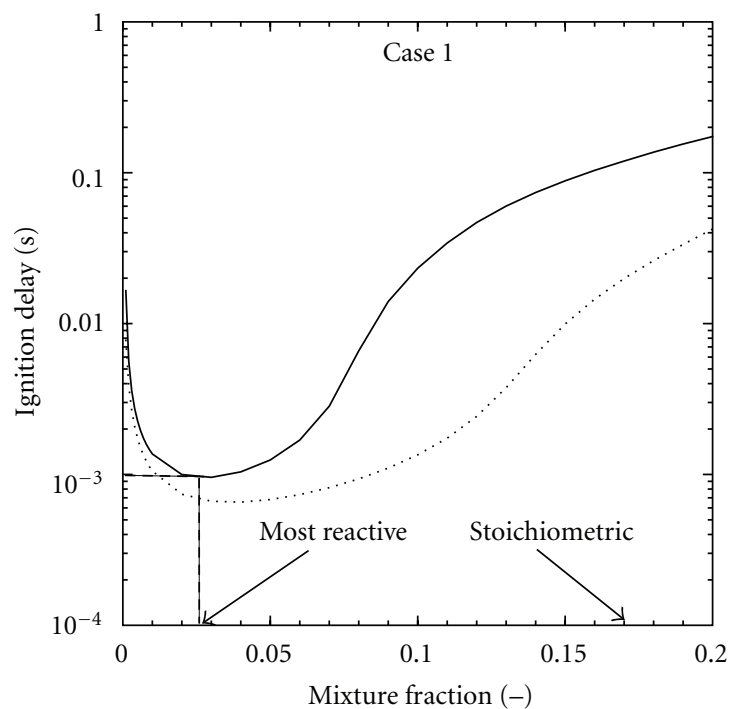

(b)

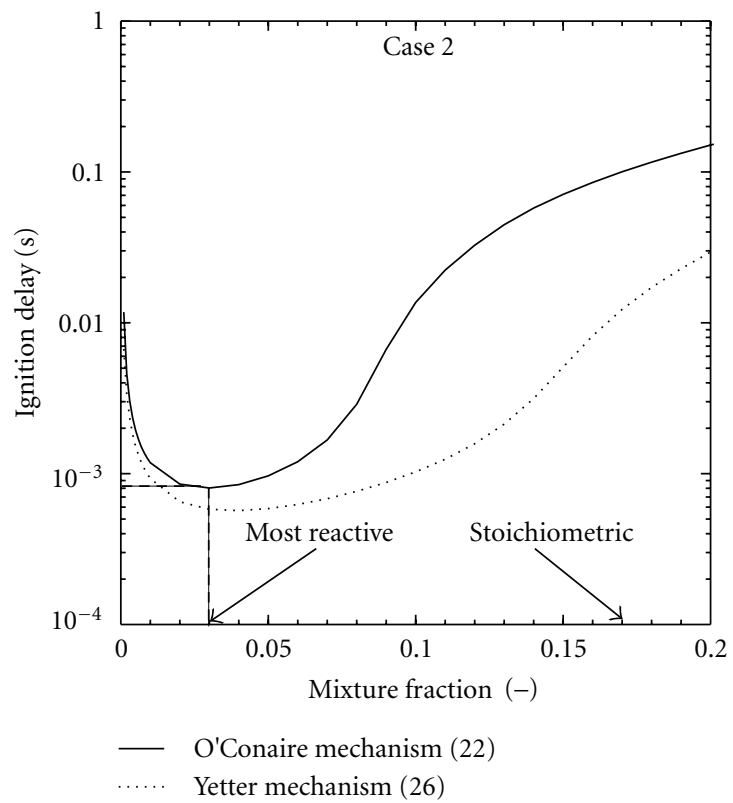

(d)

Figure 3: Most reactive mixture fraction based on ignition delay times predicted by different mechanisms for the cases described in Table 1.

Figure $4(\mathrm{~b})$ shows the time averaged axial distribution of the resolved mixture fraction. No significant difference between the curves for the two meshes is observed. This shows that there is no further refinement necessary and the Mesh 1 is a good quality LES mesh for the test case. Due to this reason, the relatively coarser Mesh 1 is used for the model validation in the following sections.

5.2.2. Mixing Field. The time averaged resolved mixture fraction and scalar dissipation rate distribution along the axis of the flow are shown in Figure 5. The time average was taken over $25 \mathrm{~ms}$ of the simulation run, which corresponds to about 5 residence times $\left(L / U_{\text {bulk }}\right)$ of the flow. Although the mixture fraction distribution does depend to some extent on coflow temperature and the heat release distribution, only one case (Case 0 ) is shown here for clarity.

According to Taylor's diffusion theory [30], the mean mixture fraction profile obeys an $x^{-2}$ power law at short distances from the nozzle and $x^{-1}$ power law at larger distances. Figure 5(b) shows that the mixture fraction decay along the axial direction $x$ at longer distances from the injector obeys an $x^{-2.2}$ power law. Taylor's law is applicable to the low 


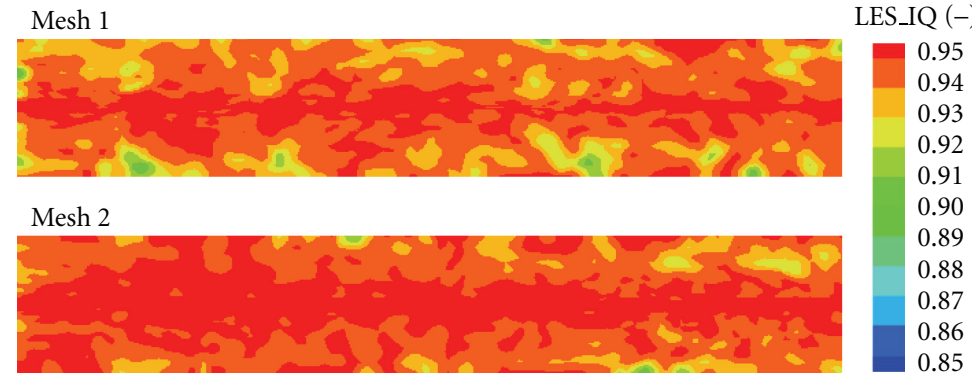

(a)

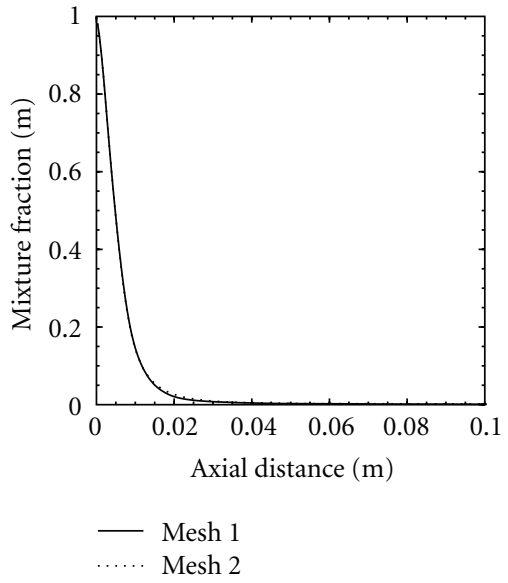

(b)

FIGURE 4: LES quality criteria (a) and the time averaged resolved axial mixture fraction distribution (b) Mesh 1: $150 \times 70 \times 48$ and Mesh 2: $200 \times 90 \times 56$ nodes in axial, radial, and azimuthal directions, respectively.

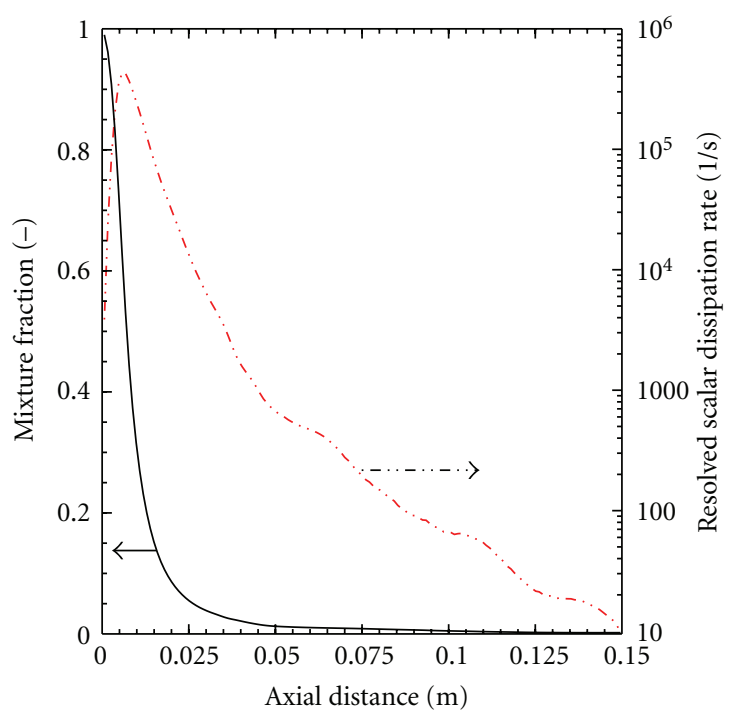

(a)

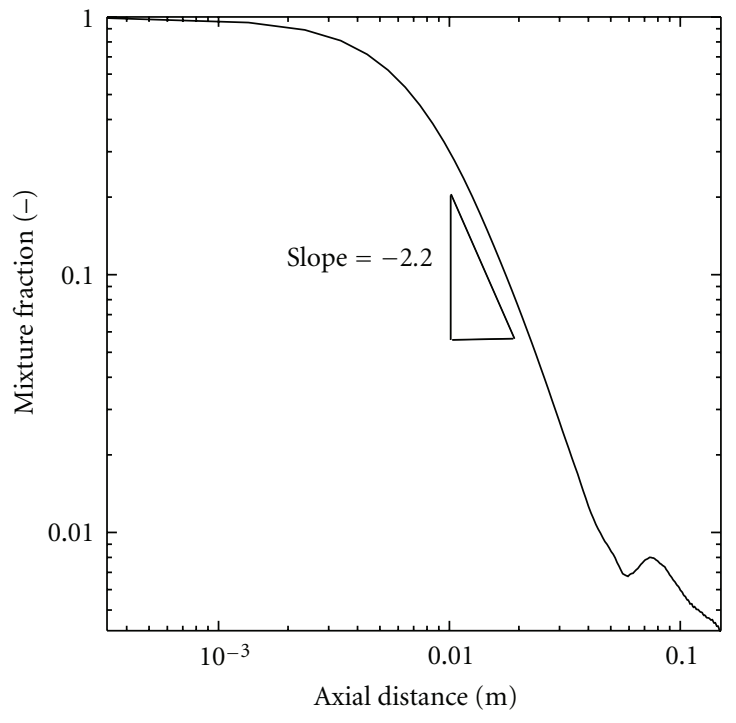

(b)

FIGURE 5: Time averaged resolved mixture fraction and scalar dissipation rate.

turbulent intensities, while the test case has a high turbulent intensity of $14 \%$, which accounts for the discrepancy.

5.2.3. Autoignition Location and Regimes. In this section the autoignition length and the subsequent kernel behavior will be compared with the experimental measurements. The criterion for autoignition used for LES is $10 \%$ rise in the value of the normalized progress variable. Figure 6 shows the mean autoignition length from the experimental measurements and the LES as a function of the coflow temperature. The mean autoignition length was determined by taking time averaged progress variable distribution over $25 \mathrm{~ms}$. The method is described later in this section in details. The reference line indicates the minimum autoignition length, calculated simply from mean bulk velocity and ignition delay at the most reactive mixture fraction

$$
l_{\text {ign,ref }}=U_{\text {bulk }} * t_{\text {ign,MR }} .
$$

The ignition delays for the most reactive mixture fraction are read from Figure 3.

The reference ignition length $l_{\text {ign,ref }}$ only considers the convective effect of the transport. The autoignition length in a turbulent flow is expected to be greater than this value. The experimental measurements (empty circles) show that the autoignition length decreases with increase in the coflow temperature. This is due to the increased reaction rates 


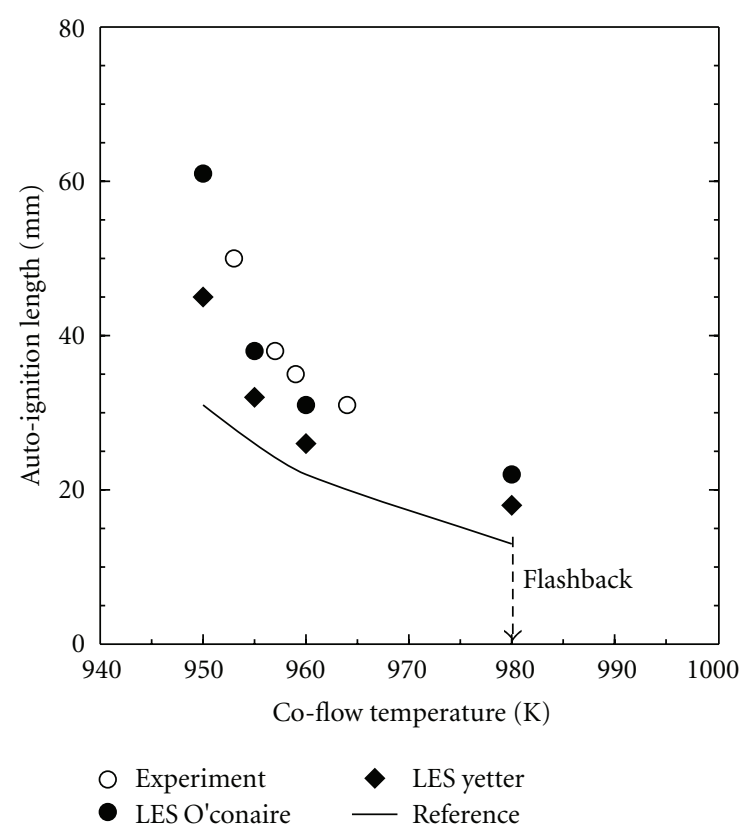

FIGURE 6: LES autoignition locations. Reference: minimum autoignition length $\left(l_{\text {ign,ref }}\right)$ using Ó Conaire mechanism [22].

at high temperatures. The filled circles are the location of autoignition in LES using the Ó Conaire mechanism. The diamonds show the same results with the Yetter mechanism. As expected from the OD PFR calculations, the autoignition length in LES predicted by the Yetter mechanism is shorter.

As the results with Ó Conaire mechanism are closer to the experimental values, the results with Ó Conaire mechanism will be described from this point.

Apart from the location of autoignition, different behavior was observed depending on the coflow temperatures. The first three cases, namely, $950 \mathrm{~K}, 955 \mathrm{~K}$, and $960 \mathrm{~K}$, show a "random spots" behavior. In this case, the axial diffusionreaction upstream cannot overcome the convective transport downstream and eventually the autoignition kernel is transported out of the domain. For the last case, the diffusionreaction transport overcomes the convective transport and the flame flashes back, resulting in an attached flame. Figure 7(a) shows a snapshot of the temperature for the four cases. The isolines show the most reactive $(0.025)$ and the stoichiometric (0.17) mixture fraction. The stoichiometric mixture fraction is seen as the enclosed isoline at the area close to the nozzle. Further downstream, only the most reactive mixture fraction is visible. The ignition spots for the first three cases are enclosed by the most reactive mixture fraction. This shows that the autoignition takes place between the most reactive and the stoichiometric mixture fraction.

Figure 7(b) shows the mixture fraction, progress variable and the temperature contour at the moment when the first autoignition kernel appears for Case 0 . The progress variable contour is purposefully shown using a nonlinear scale in order to explain the working of the model. The bottom part of the contour shows that the progress variable starts building up as soon as the fuel and oxidizer streams come in contact. Although slow, the chemical reactions are faster around the most reactive mixture fraction region. Due to convective and diffusive transport (large scalar dissipation rate), the progress variable growth is effectively retarded. During this phase, the radical pool development is emulated by the intermediate species $\mathrm{HO}_{2}$ considered in the definition of the progress variable. There is no change in the temperature observed in those regions. The autoignition takes place at the location where the convection, diffusion, and reaction terms in the transport equation of the progress variable balance. It is also to be noted that in the preautoignition regions, where the progress variable has low values, the gradients of the mixture fraction are larger than those of the progress variable. This observation validates the assumption of negligible conditioned fluctuations of the progress variable, at least in the induction region.

An important observation during the LES of $25 \mathrm{~ms}$ was that ignition spots occurred more frequently with increasing temperature. This was also observed in the experiments. LES were also carried out for coflow temperatures lower than $950 \mathrm{~K}$. It was found that at temperatures below $940 \mathrm{~K}$, no ignition was observed. Although the reaction progress variable reached values up to $Y_{c}=0.001$, it never reached the ignition point. The snapshot for Case 3 in Figure 7(b) shows a diffusion flame, which was established within the first residence time $(5 \mathrm{~ms})$ of the simulation. The autoignition took place at $22 \mathrm{~mm}$ from the injector, which is longer than the reference ignition length of $13 \mathrm{~mm}$. The flashback and diffusion flame was also observed experimentally for $980 \mathrm{~K}$.

Figure 8 shows the time averaged resolved temperature and the progress variable. The isolines show the stoichiometric and the most reactive mixture fractions. The difference in the mixture fraction distribution is due to the effect of heat release on the flow. It is interesting to observe from Figures 7 and 8 that although the autoignition kernels did not appear at the axis, the time averaged mean location lies very close the axis. This makes the determination of the mean autoignition location possible on the axis.

Figure 9 shows the time averaged resolved progress variable and the temperature along the axis of the tube. The autoignition criteria, defined as $10 \%$ rise in the progress variable, is also shown. The progress variable curves show a conspicuous rise, which makes the precise location of the autoignition possible. This increase was also observed in the experiments for the time averaged $\mathrm{OH}$ chemiluminescence images [4]. The LES autoignition lengths are close to the measured ones, as shown in Figure 6.

To summarize the results, the autoignition location and the subsequent development (extinction, stabilization, and flashback) were predicted satisfactorily by the progress variable/LES approach. In particular, with reduced coflow temperature, ignition kernels occurred less frequently. The two detailed chemical kinetic mechanisms tested, namely, the Yetter and the Ó Conaire mechanisms, showed a significant difference in the autoignition location. In this study, Ó Conaire mechanism made possible a more accurate prediction 

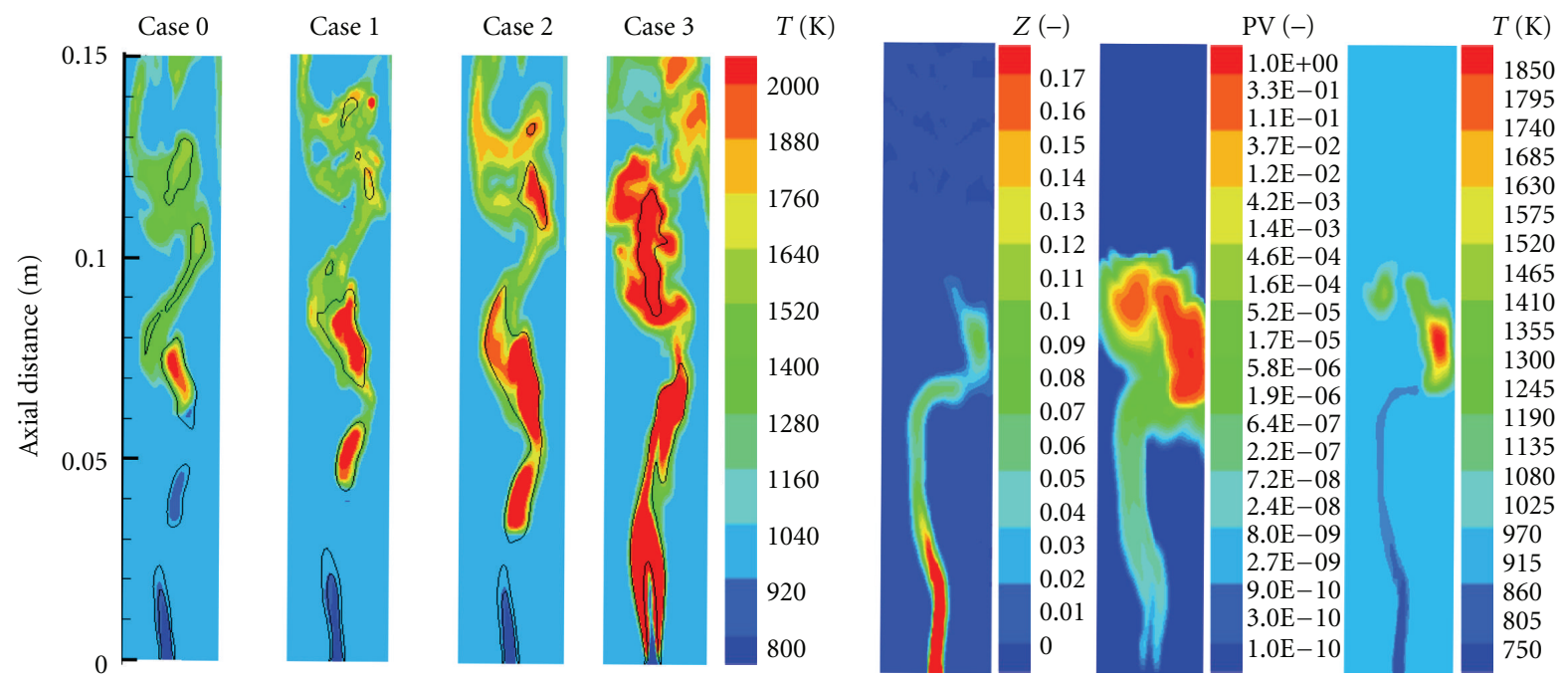

FIgURE 7: (a): Snapshot of temperature. (Lines: stoichiometric $\left(Z_{\mathrm{st}}=0.17\right)$ and most reactive $\left(Z_{\mathrm{MR}}=0.025\right)$ mixture fraction). (b): the mixture fraction $(Z)$, the normalized progress variable $(P V)$, and the temperature $(T)$ for Case 0 .
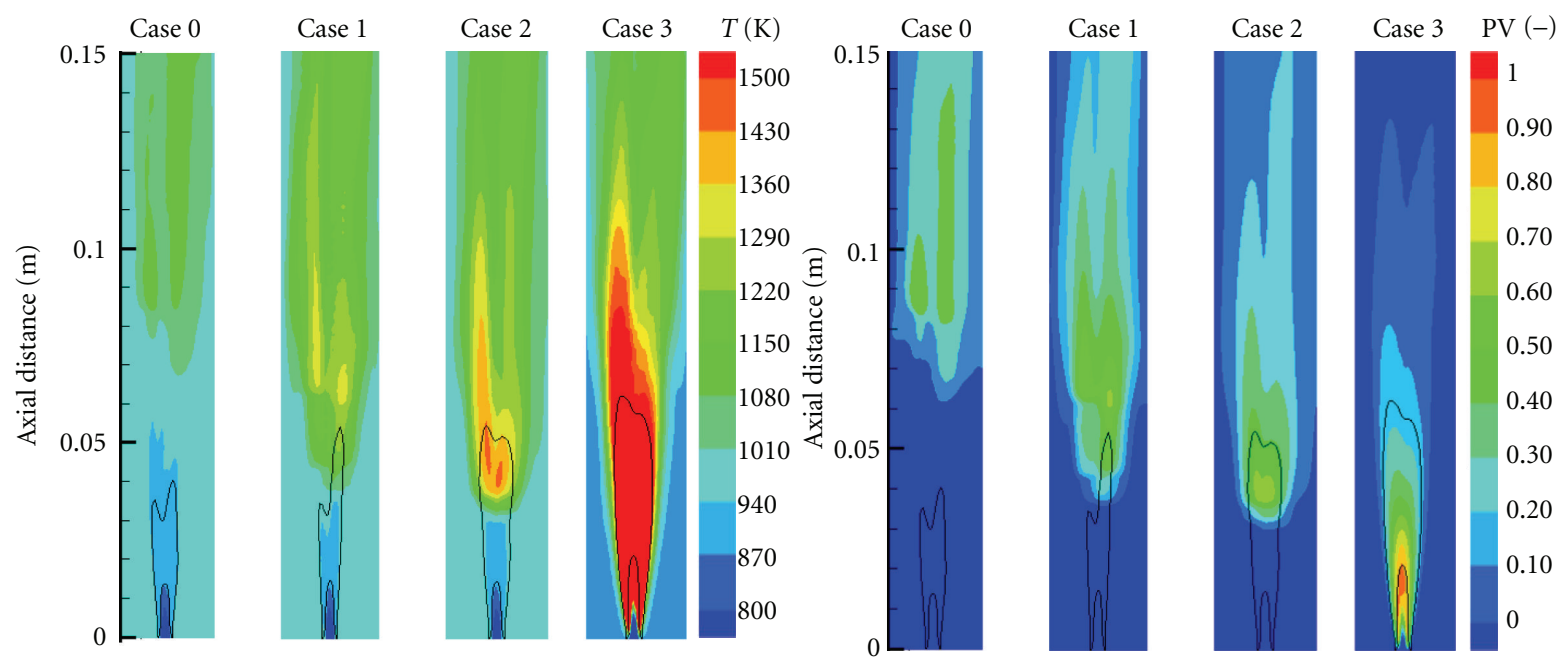

FIgURE 8: Time averaged contours of the resolved temperature (a) and the normalized progress variable PV (b). (Lines: stoichiometric $\left(Z_{\mathrm{st}}=0.17\right)$ and most reactive $\left(Z_{\mathrm{MR}}=0.025\right)$ mixture fraction $)$.

of autoignition and subsequent flame development than the Yetter mechanism.

\section{Conclusion}

A model for autoignition and heat release in turbulent flow was formulated and validated against experimental data of a hydrogen jet issuing into turbulent coflow of hot air. The model is based on a single-composite progress variable, which tracks both autoignition and heat release. Detailed chemistry based on PFR calculations is tabulated as a function of mixture fraction and progress variable. A beta function PDF is used for closure of the filtered source term for the progress variable. Fluctuations of the progress variable conditioned on the mixture fraction are neglected, as are differential and preferential diffusion effects.

The strong effect of the coflow temperature on the autoignition location and the different autoignition regimes are captured by the model. The chemical mechanism used to tabulate the chemistry is found to play a significant role in predicting the autoignition location correctly. If the mechanism of O Conaire et al. [22] is used, the mean location of autoignition in the random ignition spots regime was found to agree quantitatively with the experimental values. The flashback regime, observed experimentally at high temperatures, was also captured by the model. Compared to models using detailed chemistry and CMC or stochastic fields, significant reduction in computational costs can be realized with the progress variable/presumed PDF formulation. 


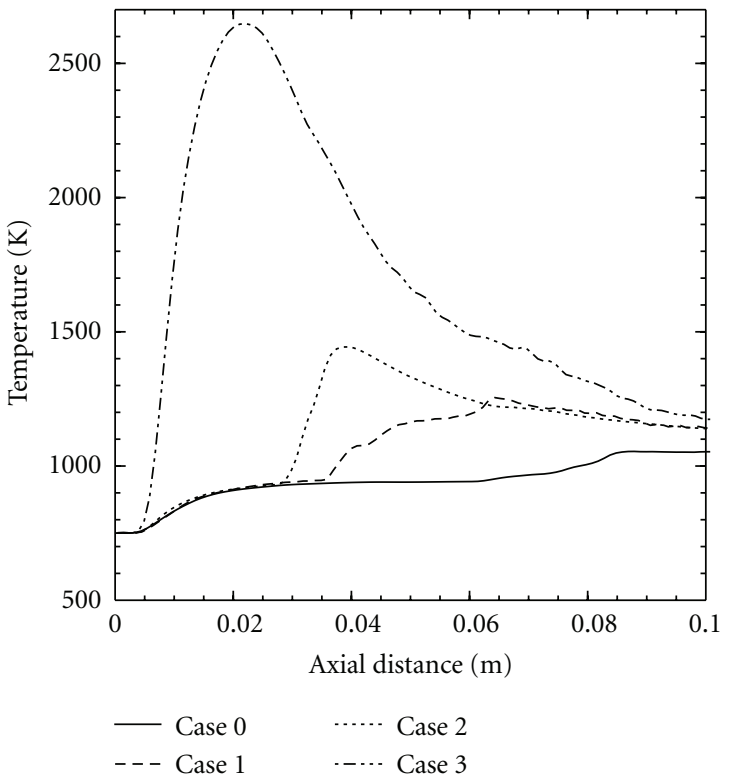

(a)

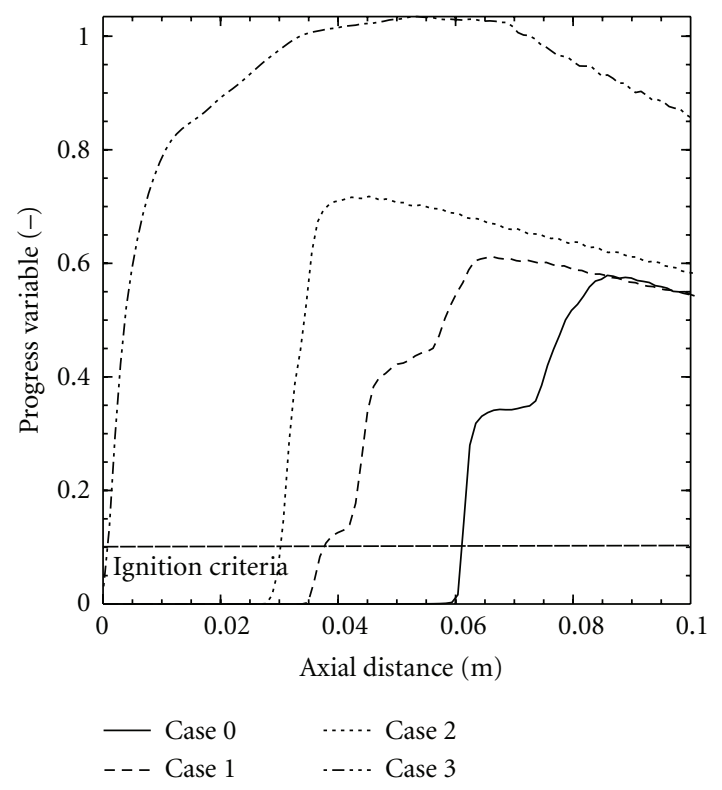

(b)

FIGURE 9: Time averaged resolved temperature (a) and the normalized progress variable PV (b) along the axis of the tube.

\section{Acknowledgments}

Financial support for this work has been provided by Alstom Power, coordinated by Uwe Ruedel. Fruitful discussions with Birute Binkute, Michael Düsing, Fernando Biagioli, and Bruno Schuermans (Alstom), and Patrick Jenny (ETH Zürich) are gratefully acknowledged.

\section{References}

[1] E. Mastorakos, "Ignition of turbulent non-premixed flames," Progress in Energy and Combustion Science, vol. 35, no. 1, pp. 57-97, 2009.
[2] F. Joos, P. Brunner, B. Schulte-Werning, K. Syed, and A. Eroglu, "Development of the sequential combustion system for the ABB GT24/GT26 gas turbine family," in Proceedings of the International Gas Turbine and Aeroengine Congress \& Exposition, ASME (96-GT-315), Birmingham, UK, 1996.

[3] M. Brandt, W. Polifke, B. Ivancic, P. Flohr, and B. Paikert, "Auto-ignition in a gas turbine burner at elevated temperature," in Proceedings of the International Gas Turbine and Aeroengine Congress \& Exposition, ASME (2003-GT-38224), Atlanta, Ga, USA, 2003.

[4] C. N. Markides and E. Mastorakos, "An experimental study of hydrogen autoignition in a turbulent co-flow of heated air," Proceedings of the Combustion Institute, vol. 30, no. 1, pp. 883891.

[5] E. Mastorakos, C. N. Markides, and Y. M. Wright, "Hydrogen auto-ignition in a turbulentduct flow: experiments and modeling," in Proceedings of the 12th Conference on Modeling Fluid Flow (CMFF'03), 2003.

[6] I. Stanković, A. Triantafyllidis, E. Mastorakos, C. Lacor, and B. Merci, "Simulation of hydrogen auto-ignition in a turbulent Co-flow of heated air with LES and CMC approach," Flow, Turbulence and Combustion, vol. 86, no. 3-4, pp. 689-710, 2011.

[7] S. Navarro-Martinez and W. P. Jones, "Study of hydrogen auto-ignition in a turbulent air co-flow using a Large Eddy Simulation approach," Computers and Fluids, vol. 37, no. 7, pp. 802-808, 2008.

[8] L. Vervisch, R. Hauguel, P. Domingo, and M. Rullaud, "Three facets of turbulent combustion modelling: DNS of premixed V-flame, LES of lifted nonpremixed flame and RANS of jetflame," Journal of Turbulence, vol. 5, no. 1, 2004.

[9] C. D. Pierce, Progress-variable approach for large-eddy simulation of turbulent combustion, Ph.D. thesis, Stanford University, Stanford, Calif, USA, 2001.

[10] C. D. Pierce and P. Moin, "Progress-variable approach for large-eddy simulation of non-premixed turbulent combustion," Journal of Fluid Mechanics, no. 504, pp. 73-97, 2004.

[11] P. Domingo, L. Vervisch, and K. Bray, "Partially premixed flamelets in LES of nonpremixed turbulent combustion," Combustion Theory and Modelling, vol. 6, no. 4, pp. 529-551, 2002.

[12] P. Domingo, L. Vervisch, and D. Veynante, "Auto-ignition and ame Propagation effects in LESof burned gases diluted turbulent combustion," in Proceedings of the Summer Program, pp. 337-348, Center for Turbulence Research, 2006.

[13] J. Galpin, C. Angelberger, A. Naudin, and L. Vervisch, "Largeeddy simulation of $\mathrm{H} 2$-air auto-ignition using tabulated detailed chemistry," Journal of Turbulence, vol. 9, no. 13, pp. $1-21,2008$.

[14] D. Bradley, L. K. Kwa, A. K. C. Lau, M. Missaghi, and S. B. Chin, "Laminar flamelet modeling of recirculating premixed methane and propane-air combustion," Combustion and Flame, vol. 71, no. 2, pp. 109-122, 1988.

[15] D. Bradley and A. K. C. Lau, "The mathematical modelling of premixed turbulent combustion," Pure and Applied Chemistry, vol. 62, no. 5, pp. 803-814, 1990.

[16] A. C. Benim and K. J. Syed, "Laminar flamelet modelling of turbulent premixed combustion," Applied Mathematical Modelling, vol. 22, no. 1-2, pp. 113-136, 1998.

[17] W. Polifke, M. Bettelini, W. Geng, U. C. Muller, W. Weisenstein, and P. A. Jansohn, Comparisonof Combustion Models for Industrial Applications, Eccomas 98, Athens, Greece, 1998.

[18] C. S. Chang, Y. Zhang, K. N. C. Bray, and B. Rogg, "Modelling and simulation of autoignition under simulated diesel-engine 
conditions," Combustion Science and Technology, vol. 113-114, pp. 205-219, 1996.

[19] L. P. H. De Goey and J. H. M. Ten Thije Boonkkamp, "A flamelet description of premixed laminar flames and the relation with flame stretch," Combustion and Flame, vol. 119, no. 3, pp. 253-271, 1999.

[20] B. Ivancic, P. Flohr, B. Paikert, M. Brandt, and W. Polifke, "Auto-ignition and heat release in a gas turbine burner at elevated temperature," in Proceedings of the International Gas Turbine and AeroengineCongress \& Exposition, ASME GT2004-53339, Vienna, Austria, 2004.

[21] M. Brandt, W. Polifke, and P. Flohr, "Approximation of joint PDFs by discrete distributions generated with Monte Carlo methods," Combustion Theory and Modelling, vol. 10, no. 4, pp. 535-558, 2006.

[22] M. Ó Conaire, H. J. Curran, J. M. Simmie, W. J. Pitz, and C. K. Westbrook, "A comprehensive modeling study of hydrogen oxidation," International Journal of Chemical Kinetics, vol. 36, no. 11, pp. 603-622, 2004.

[23] O. Gicquel, N. Darabiha, and D. Thévenin, "Laminar premixed hydrogen/air counterflow flame simulations using flame prolongation of ILDM with differential diffusion," Proceedings of the Combustion Institute, vol. 28, no. 2, pp. 19011907, 2000.

[24] C. D. Pierce and P. Moin, "A dynamic model for subgrid-scale variance and dissipation rate of a conserved scalar," Physics of Fluids, vol. 10, no. 12, pp. 3041-3044, 1998.

[25] ANSYS FLUENT Academic Research, Release 6.3, 2006.

[26] R. A. Yetter, F. L. Dryer, and H. Rabitz, "A comprehensive reaction mechanism for carbonmonoxide/ hydrogen/oxygen kinetics," Combustion Science and Technology, vol. 79, pp. 97129, 1991.

[27] Caltech, "Cantera: object-oriented software for reacting," Release 1.8.0, 2009, http://code.google.com/p/cantera/.

[28] J. Li, Z. Zhao, A. Kazakov, and F. L. Dryer, "An updated comprehensive kinetic model of hydrogen combustion," International Journal of Chemical Kinetics, vol. 36, no. 10, pp. 566-575, 2004.

[29] I. B. Celik, Z. N. Cehreli, and I. Yavuz, "Index of resolution quality for large eddy simulations," Journal of Fluids Engineering, vol. 127, no. 5, pp. 949-958, 2005.

[30] G. Taylor, "Dispersion of soluble matter in solvent flowing slowly through a tube," Proceedings of the Royal Society A, vol. 219, pp. 186-203, 1953. 

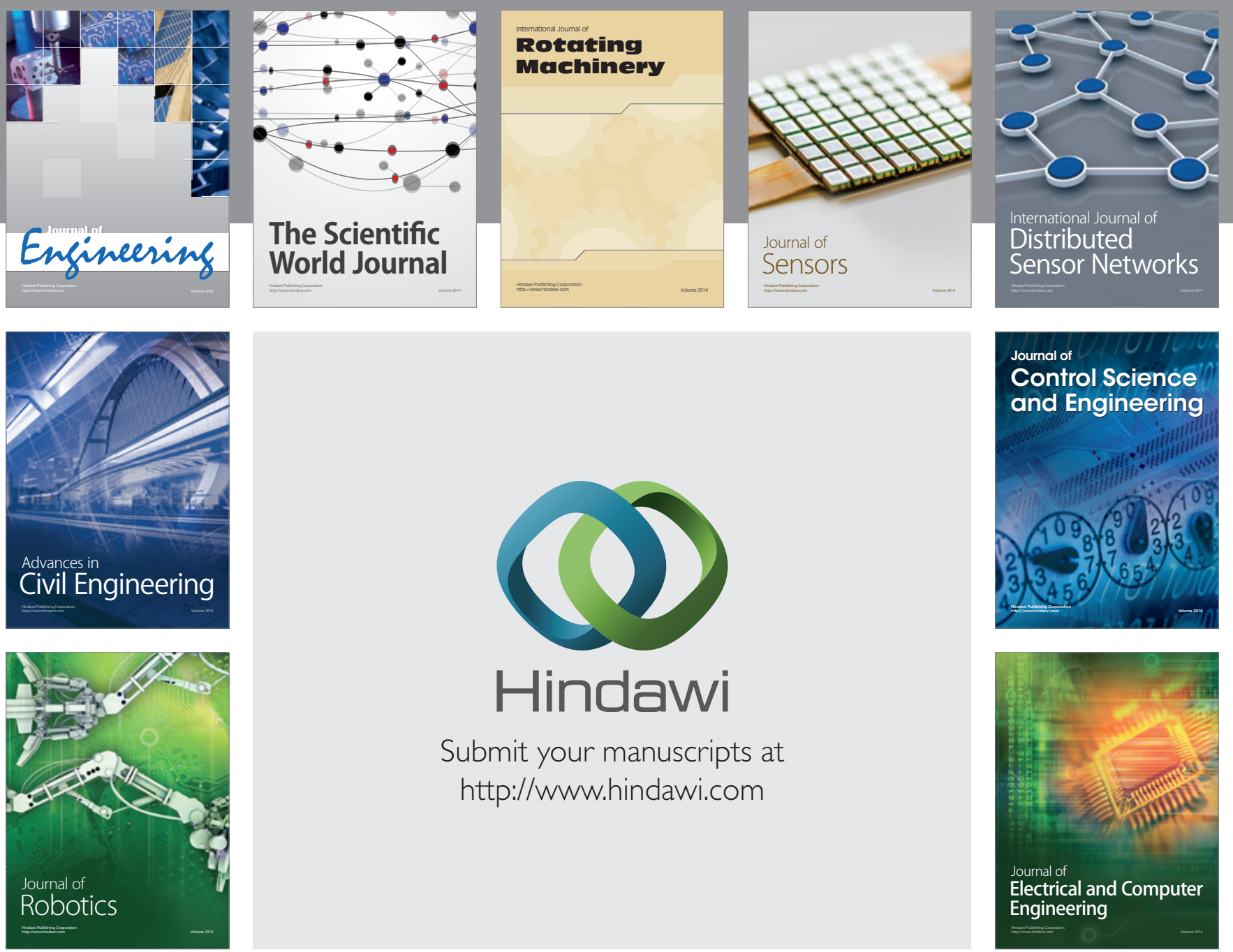

Submit your manuscripts at

http://www.hindawi.com
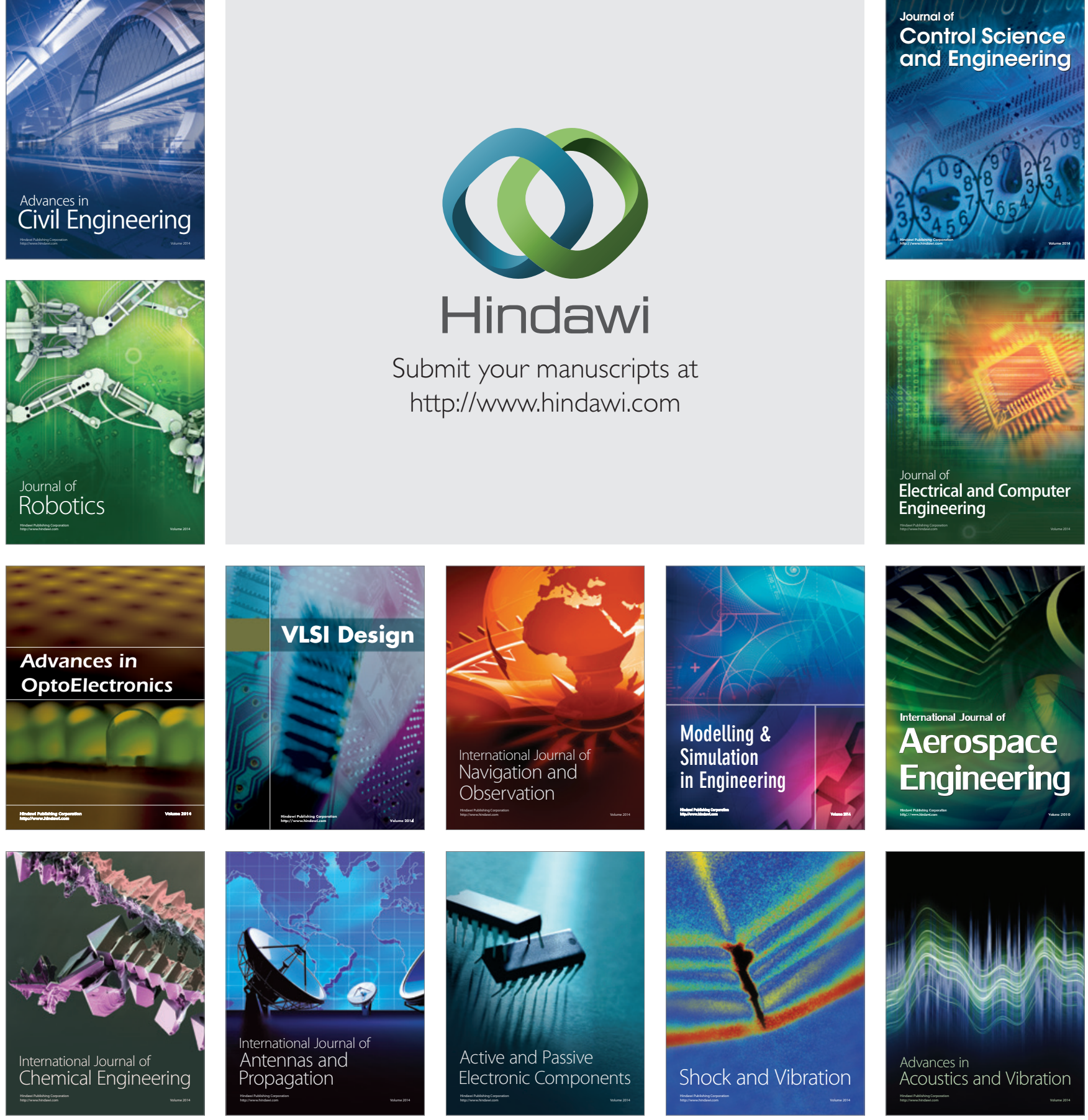